\title{
METHYLPHENIDAT: EIN RÜCKBLICK
}

\section{ÜBERPRÜFUNG ARTIKEL}

OLIVEIRA, Vinicius Faustino Lima de ${ }^{1}$, MOREIRA, Danilo José Silva², FONSECA, Juliana Brito da ${ }^{3}$, ROSSI, Karoline ${ }^{4}$, VASCONCELOS, Suzana dos Santos ${ }^{5}$, DIAS, Claudio Alberto Gellis de Mattos ${ }^{6}$, OLIVEIRA, Euzébio de ${ }^{7}$, DENDASCK, Carla Viana ${ }^{8}$, ARAÚJO, Maria Helena Mendonça de ${ }^{9}$ FECURY, Amanda Alves ${ }^{10}$

OLIVEIRA, Vinicius Faustino Lima de. Et al. Methylphenidat: ein rückblick. Revista Científica Multidisciplinar Núcleo do Conhecimento. Jahrgang 06, Ed. 05, Vol. 01, S. 05-14. Mai 2021. ISSN: 2448-0959, Zugriffsverbindung:

https://www.nucleodoconhecimento.com.br/gesundheit/methylphenidat-einrueckblick, DOI: 10.32749/nucleodoconhecimento.com.br/gesundheit/methylphenidat-ein-rueckblick

\section{ZUSAMMENFASSUNG}

Methylphenidat (MFD) ist ein Amphetamin-abgeleitetes Medikament, das das Zentrale Nervensystem (SNC) stimuliert und erhöhte Aufmerksamkeit, weniger Ablenkung und erhöhtes Gefühl der Motivation fördert. Diese Studie zielt darauf ab, die wichtigsten

\footnotetext{
${ }^{1}$ Mediziner. Föderale Universität von Amapá (UNIFAP).

${ }^{2}$ Mediziner. Föderale Universität von Amapá (UNIFAP).

${ }^{3}$ Medizinische Akademiker. Föderale Universität von Amapá (UNIFAP).

${ }^{4}$ Medizinische Akademiker. Föderale Universität von Amapá (UNIFAP).

${ }^{5}$ Medizinische Akademiker. Föderale Universität von Amapá (UNIFAP).

${ }^{6}$ Biologe, PhD in Theorie- und Verhaltensforschung, Professor und Forscher des Chemie-Studiengangs des Instituts für Grund-, Technik- und Technologiepädagogik (IFAP) und des Graduate Program in Professional and Technological Education (PROFEPT IFAP).

${ }^{7}$ Biologe, PhD in Tropenkrankheiten, Professor und Forscher des Physical Education Course, Federal University of Pará (UFPA).

${ }^{8}$ Theologe, PhD in Klinischer Psychoanalyse. Er arbeitet seit 15 Jahren mit wissenschaftlicher Methodik (Forschungsmethode) in der wissenschaftlichen Produktionsberatung für Master- und Doktoranden. Spezialist für Markt- und Gesundheitsforschung, Doktorand in Kommunikation und Semiotik (PUC SP).

${ }^{9}$ Doktor, Master in Lehr- und Gesundheitswissenschaften, Professor und Forscher des Medical Course of Macapá Campus, Federal University of Amapá (UNIFAP).

${ }^{10}$ Biomedizin, PhD in Tropenkrankheiten, Professor und Forscher des Medical Course of Macapa Campus, Federal University of Amapá (UNIFAP), Pro-Rektor of Research and Graduate Studies (PROPESPG) der Föderalen Universität von Amapá (UNIFAP).

RC: 84391

Zugriffsverbindung:
} 
pharmazeutischen Eigenschaften von MFD zu beschreiben, die klinischen Indikationen für die Verwendung von MFD hervorzuheben und die möglichen unerwünschten Ereignisse, die sich aus dem Verbrauch von MFD ergeben, zu beschreiben. Dazu haben wir in den Datenbanken ScienceResearch.com, SciELO, LILACS, PubMed und EMBASE sowie in den institutionellen Repositories der Föderalen Universität von São Paulo, der Föderalen Universität Minas Gerais und der Föderalen Universität Ceará unter Verwendung der Schlüsselwörter und Assoziationen "Methylphenidat", "Ritalin", "Effekte" und "effects" recherchiert. Es wurde nachgewiesen, dass das Medikament aus einer racemischen Mischung besteht, die aus vier Stereoisomen gebildet wird, und der pharmakologisch aktive Wirkstoff ist Racemate Dextrogyl (d,I)-treo-MFD. Es wirkt auf die Blockade von Dopamin und Noradrenalin-Transporter aus dem ZNS, Förderung von Effekten, die seine Verschreibung rechtfertigen, um Aufmerksamkeitsdefizit-Hyperaktivitäts-Störung (TDAH) zu behandeln. In den letzten Jahren hat der Konsum für nichttherapeutische Zwecke zugenommen. Einige Nebenwirkungen seiner Verwendung wurden berichtet, und Schlaflosigkeit, Reizbarkeit und Kopfschmerzen wurden berichtet.

Schlüsselwörter: Methylphenidat, Dopamin, Noradrenalin.

\section{EINLEITUNG}

Methylphenidat (MFD) ist ein Stimulator des Zentralnervensystems (SNC) aus Piperidin, einer Art Amphetamin (PAPA, 2013). Die MFD hat einen höheren Verbrauch als die Summe aller anderen Psychostimulanzien, da sie die am meisten konsumierte in der Welt ist (SALVIANO, 2015).

In den frühen 1990er Jahren wurden weltweit 2,8 Tonnen MFD produziert. Im Jahr 2000 wurde dieser Wert auf 16 Tonnen erhöht, zehn Jahre später erreichte die statistische Marke 43 Tonnen. In Brasilien im Jahr 2011413 kg MFD (CALIMAN; DOMITROVIC, 2013). 
Es wird für Personen mit Aufmerksamkeitsdefizit-Hyperaktivitätsstörung (TDAH) und für Narkolepsie (CALDEIRA, 2015) verschrieben. In Brasilien ist MFD unter den Namen Ritalina $\AA$, Ritalina $L A \circledast$ und Concerta $\AA$ erhältlich, alle mit oraler Aufnahme. Die MFD-Freisetzungsdynamik im Körper hat sofortige und langsame Handlungsoptionen, wie Ritalin $\AA$ die sofortige Wirkung hat, während Ritalin LA® und Concerta $\AA^{\circledR}$ lang wirkende Formeln präsentieren (BATISTA, 2015).

Bei Beginn der Behandlung mit MFD reichen die Für säuglingspatienten angegebenen Dosen zwischen 5 und $20 \mathrm{mg} / \mathrm{Tag}$, mit möglichen Anpassungen, und Inkremente können bis zur maximalen Tagesdosis von $60 \mathrm{mg} / \mathrm{Tag}$ vorgenommen werden. Eine weitere Möglichkeit zur Bestimmung der Tagesdosis besteht darin, mit zwei Tagesdosis von 0,3 mg/kg zu beginnen, und je nach Bedarf kann eine Erhöhung um $0,1 \mathrm{mg} / \mathrm{kg} /$ Dosis wöchentlich erfolgen und sollte $2 \mathrm{mg} / \mathrm{kg} / \mathrm{Tag}$ nicht überschreiten. Aufgrund des Mangels an Studien an Kindern unter 6 Jahren ist die Verwendung von MFD in dieser Population nicht angegeben. Bei Erwachsenen liegt die Tagesdosis von MFD zwischen 10 und 72mg (FARIA, 2017).

Die MFD sorgt für mehr Aufmerksamkeit, was zu weniger Ablenkung und erhöhtem Motivationsgefühl führt, was zu Interesse am Erreichen von Zielen und Aktionen führt (CÂNDIDO, 2018). In Brasilien dient die legale Verwendung von MFD nur der Behandlung von Krankheiten und Störungen. Dieses Medikament gehört zur Liste A3 von Psychopharmaka abhängig von besonderer Kontrolle, und seine Anschaffung ist auf die spezielle gelbe Rezeptur und Die Beibehaltung des Rezepts in der Apotheke zum Zeitpunkt des Verkaufs konditioniert (FARIA, 2017).

\section{ZIELE}

Beschreiben Sie die wichtigsten pharmazeutischen Eigenschaften von MFD. Markieren Sie die klinischen Indikationen für die Anwendung von MFD. Erläutern Sie die möglichen unerwünschten Ereignisse, die sich aus dem Verbrauch von MFD ergeben. 


\section{METHODEN}

Die verwendeten Referenzen wurden durch Forschunginder in den Datenbanken erhalten: ScienceResearch.com, SciELO, LILACS, PubMed, EMBASE, Institutional Repository der Föderalen Universität von São Paulo, Institutionelles Repository der Föderalen Universität Minas Gerais und Institutionelles Repository der Föderalen Universität Ceará, unter Verwendung der folgenden Schlüsselwörter und Assoziationen: "methylphenidat", "ritalin", "Effekte" und "effects".

Die Methode zur Auswahl der Papiere war: Identifikation, Screening, Förderfähigkeit und Inklusion. Um in diese Überprüfung einbezogen zu werden, mussten die Studien über einen vollständigen Text verfügen, der in den untersuchten Datenbanken zugänglich ist, mit relevanten Inhalten in Bezug auf die MFD, sowie in einer der folgenden Sprachen verfügbar sein: Portugiesisch, Englisch oder Spanisch. Ausschließlich in der PubMed-Plattform wurde ein zeitlicher Filter verwendet, in dem die Publikationen der letzten 5 Jahre verwendet wurden, zusätzlich zu Arbeiten im Zusammenhang mit Menschen.

Die Identifizierung war das Ende des Treffens von Studien, die in der Literatur über MFD in den Datenbanken existieren. Das Screening erfolgte nach einer kurzen Analyse des Titels und der Zusammenfassung der Veröffentlichungen, wodurch diese Studien in die Kategorie der förderfähigen Studien erhoben wurden. Letztendlich wurden alle förderfähigen Werke vollständig gelesen und folglich diejenigen ausgeschlossen, die nicht mit den Zielen dieser Überprüfung in Einklang standen und in den vorherigen Phasen vielleicht nicht ausgeschlossen wurden. Schließlich wurden die genehmigten in alle früheren Phasen der angewandten Methode einbezogen.

\section{ERGEBNISSE}

Die Forschung über MFD in den Datenbanken führte zu 1038 Artikeln in der methodischen Phase der Identifizierung. Beim Screening wurden 981 nicht durchgeführt, da nur 5,49 \% der Studien förderfähig waren. Schließlich wurden bei 
vollständiger Lesart aller förderfähigen Papiere 37 Papiere aufgenommen und 35,09 $\%$ der förderfähigen Papiere verworfen.

\section{MFD PHARMAKOLOGIE}

Die MFD, deren IUPAC (International Union of Pure and Applied Chemistry) Nomenklatur Metil 2-fenil-2(piperidil) Acetatist ist, hat zwei chirale Kohlenstoffe und die chemische Formel $\mathrm{C}_{14} \mathrm{H}_{19} \mathrm{NO}_{2}$. Es besteht aus einer racemischen Mischung aus vier Stereoisomen, die $d$-treo-MFD, I-treo-MFD, $d$-eritro-MFD und l-eritro-MFD sind. Der pharmakologisch aktive Wirkstoff ist Racemate Dextrogyl (d,I)-treo-MFD, das mit Dopaminrezeptoren im gestreiften Körper reagiert und noradrerge Stimulation im Kortex bietet (BATISTA, 2015; FREESE et al., 2012; LINHARES, 2012).

MFD ist ein indirekter Agonist von Dopamin und Noradrenalin, intensiv beeinflussen die Wirkung dieser Neurotransmitter in Motorzentren und Aufmerksamkeit (BATISTA, 2015; BATISTELA, 2011). Es wirkt durch hemmung der Dopamin-Wiederaufnahme durch präsinaptische Neuronen von kortikalen und subkortikalen Regionen (FREESE et al., 2012).

\section{THERAPEUTISCHE ANWENDUNGEN VON MFD}

MFD ist das erste Psychostimulans für den pharmakologischen Ansatz für ADHS und für die Behandlung von Narkolepsie, einer sehr seltenen Schlafstörung. Es wird auch für Freizeit-, ästhetische und kognitive verbesserte Zwecke im Zusammenhang mit erhöhter Schul- und Arbeitsproduktivität (BARROS; ORTEGA, 2011; BATISTELA, 2011; BRANT; CARVALHO, 2012; CALDEIRA, 2015; CÂNDIDO, 2018; SALVIANO, 2015).

Es wird berichtet, dass die Assoziation von MFD mit selektivem Serotonin-RecaptureInhibitor (ISRS) bei der Therapie der Symptome des Gilles de la Tourette-Syndroms (PASSOS; LÓPEZ, 2010), sowie für die Behandlung von kognitivem Defizit bei einem Patienten, der Opfer einer traumatischen Hirnverletzung (TCE) (ROCHA; MALLOYDINIZ; HARA, 2006).

RC: 84391

Zugriffsverbindung: 
MFD wird auch bei erwachsenen Patienten mit fortgeschrittenen Neoplasmen verwendet, um Schläfrigkeit aufgrund der Verwendung von Opioiden, depressiven Symptomen, hypoaktivem Delirium und Müdigkeit aufgrund von Krebs zu behandeln. Es gibt eine Beschreibung, dass MFD eine therapeutische Alternative für Patienten mit akuter Manie (CAMPOS et al., 2016; LÓPEZ-GARCÍA; HEGERL, 2013; PAPA, 2013).

\section{ADVERSE REAKTIONEN VON MFD USE}

Kinder und Jugendliche haben als Hauptnebenwirkungen Schlaflosigkeit und verminderten Appetit, direkt proportional die Dosen von MFD aufgenommen, neben der Darstellung von Xerostomie und Reizbarkeit. Eine schwere Verwicklung in den psychiatrischen Zustand kann auftreten, potenzieren oder verursachen psychotische Störungen oder Episoden von Manie und Tics. Effekte wie Bauchschmerzen und Kopfschmerzen sind wichtig, sowie eine kleine Erhöhung der Herzfrequenz (BATISTA, 2015; FARIA 2017; LOUZÃ; MATTOS, 2007; PASTURA; MATTOS, 2004).

Bei längerem Gebrauch, Nebenwirkungen wie Verringerung der Höhe und Entwicklung der chemischen Abhängigkeit beim Patienten (BRANT; CARVALHO, 2012; PASTURA; MATTOS, 2004). Darüber hinaus wird beobachtet, dass der Blutdruck der MFD-Nutzer um mehr als 10 mmHg erhöht werden kann (CÂNDIDO, 2018).

Eine Studie mit tierischen Meerschweinchen zeigt, dass die akute und chronische Verabreichung von MFD Demoxyribonukleinsäure (DNA) im gestreiften und Hippocampus-Körper schädigt (SALVIANO, 2015). Es gibt Berichte, dass MFD verursacht auch Übelkeit, Erbrechen Abnahme des Wachstums und Gewichtrate, Arrhythmien, Husten und Halluzinationen, die visuelle und taktile in der Natur im Zusammenhang mit Insekten, Reptilien oder Ungeziefer sind (BATISTA, 2015). Bei Kindern und jungen Erwachsenen, bei denen TDAH diagnostiziert wird, bei denen MFD angewendet wird, tritt eine Psychose bei 1 von 660 Personen auf (MORAN et al., 2019). 
Alopezie wird für die Verwendung von MFD gemeldet (NÚÑES-GARCES; SÁNCHEZGAYANGO; ROMERO-PÉREZ, 2018) und diese langsame Freisetzung von MFD kann mit der Entwicklung des Raynaud-Phänomens zusammenhängen, einer Beteiligung, die durch übermäßige Vasokonstriktion von Arteriolen gekennzeichnet ist, die die Haut bewässern (OTERO et al., 2012).

Grazina et al. (2018) beschreiben Oberschenkelfraktur bei einem Mädchen mit TDAH mit MFD behandelt diagnostiziert, Assoziieren des Medikaments als mögliche Ursache. In dieser Studie weisen sie darauf hin, dass sich die Knochendichte eines Kindes, das MFD verwendet, von der unterscheidet, die nicht verwendet wird, was darauf hindeutet, dass das Medikament das Risiko einer Fraktur erhöht.

Bei Ratten, die histologisch humankompatibles Lungengewebe haben, verursachte die Verabreichung von MFD Schäden am Lungenparenchym durch die Zerstörung von Alveolarsepta (RAPELLO et al., 2015). MFD kann als induzierender Erreger morphologischer Differenzierungen in Speicheldrüsen von Ratten angesehen werden (LIMA et al., 2016).

Der Zusammenhang von MFD mit der Entwicklung von Systemischer Sklerose wird bei Patienten mit Verdacht auf Autoimmun- und Vasopathische Erkrankung (MERIDOR; LEVY, 2019). Es gibt Berichte über den Fall eines Patienten von vier Jahrzehnten des Lebens, der bariatrische Chirurgie mit roux-ähnlichen laparoskopischen Magenbypass, der die MFD seit etwa einem Jahr verwendet hatte, und nach zwei Wochen der Operation begann Symptome der MFD-Toxizität, wie Paranoia und auditive Halluzinationen (LUDVIGSSON; HAENNI, 2016).

\section{DISKUSSION}

\section{MFD OPERATING MECHANISMUS UND ITS METABOLIZATION}

Obwohl die pharmakologischen Merkmale der MFD gut etabliert sind, ist ihr Funktionsweisemechanismus noch nicht vollständig verstanden (BRUNELLI, 2013; LINHARES, 2012).

RC: 84391

Zugriffsverbindung: 
Es ist bekannt, dass MFD wirkt auf die dopaminergen und noradrenergen Bahnen der kortikalen und subkortikalen Regionen des Gehirns, vor allem im gestreiften Körper, Bindung an Dopamin und Noradrenalin-Transporter, blockieren sie. Das Ergebnis ist eine größere Verfügbarkeit dieser in lokalen synaptischen Rissen, die erregende Effekte erzeugt, die über die Vorteile der MFD reflektieren (BATISTELA, 2011; BRUNELLI, 2013; CÂNDIDO, 2018; FERNÁNDEZ, 2018; PALHARES, 2015; SALVIANO, 2015).

Die wichtigsten etablierten Dopamin-Transporter sind DAT1 und DRD4. Es gibt einen Bericht in der Literatur, dass einige Mutationen in den Genen, die diese Transporter kodieren, mit einer erhöhten Reaktion auf MFD verbunden sind, eine Reaktion, die auch mit sozialen und klinischen Faktoren verbunden ist (BATISTA, 2015).

Der Anstieg des Dopaminspiegels aufgrund des Medikaments tritt in Bereichen des SNC auf, die anfällig für die Wirkung von Missbrauchsmedikamenten sind (FARIA, 2017).

Noradrenalin hat NAT als Transporter, Das ist der primäre Mechanismus der dopaminergen Signalweg Inaktivierung. Wenn blockiert, Es erhöht die präsinaptitischen Ebenen von Noradrenalin, was zu einer größeren Aktivierung des Noradrengy-Signalwegs führt (LINHARES, 2012).

Dopamin wirkt direkt in der Modulation der kognitiven und motorischen Funktionen, Gedächtniskonsolidierung, Aufmerksamkeitsförderung, zusätzlich zur Verarbeitung einiger Umweltinformationen von größter Bedeutung für das Überleben, Durchführung dieser Funktionen, zum Teil auf die D1 (exzitatorische Funktion) und D2 (Hemmfunktion) Rezeptoren des präfrontalen Kortex. Daher wird angenommen, dass das Defizit in seiner Funktion das Auftreten von Unaufmerksamkeit und Hyperaktivität verursacht, zwei klinische Anzeichen kompatibel mit dem Bild von TDAH (BATISTELA, 2011; LIANG et al., 2018; LINHARES, 2012; OCHI; VIEIRA, 2016).

In Bezug auf Noradrenalin, Es ist bekannt, dass es in Bereichen wirkt, die Verhaltenshemmung Reize beeinflussen. Daraus lässt sich ableiten, dass das Defizit RC: 84391

Zugriffsverbindung: 
in seiner Funktion zur Unfähigkeit führen kann, Impulse zu kontrollieren, ein weiteres Merkmal von TDAH (LIANG et al., 2018; LINHARES, 2012; OCHI; VIEIRA, 2016).

Es gibt drei MFD-Formulierungen auf dem Markt: Ritalin $\AA$, Ritalin LA® und Concerta $\mathrm{LA} \Theta$, die in Bezug auf die Halbwertszeit, Die Freigabezeit und die Dauer der Aktion variieren. Die erste besteht aus einer schnellen Wirkungsformulierung, die MFDSpitzen etwa 1 bis 3 Stunden nach der Einnahme fördert, mit einer Halbwertszeit zwischen 2 bis 3 Stunden und einer Nutzungsdauer zwischen 3 und 6 Stunden. Die beiden letztgenannten bestehen aus schnell wirkenden Formulierungen, die MFDSpitzen etwa 3 bis 4 Stunden nach der Einnahme fördern, mit einer Halbwertszeit von 4 Stunden und einer Nutzungsdauer von 8 bis 12 Stunden. Was sich von innen unterscheiden wird, ist die Menge der Dosen, die pro Tag eingenommen werden können: Ritalin LA $\circledast$ kann 1 oder 2 mal pro Tag eingenommen werden; La Concerta $\AA$ 1 Mal pro Tag (LINHARES, 2012; LOUZÃ; MATTOS, 2007; PAPA, 2013).

Die Absorption von MFD erfolgt vollständig und kann die hämatozephale Barriere aufgrund ihrer Lipolubilität leicht überqueren (LINHARES, 2012).

Etwa 48 bis 96 Stunden, MPH wird durch das hepatische mikrosomale System metabolisiert, durch einen Hydrolyse-Prozess, der Ritalinsäure erzeugt, Substrat aus dem Körper durch Harnausscheidung eliminiert. Der Rest des Medikaments, das nicht metabolisiert wird, wird zusammen mit Gallensekretion eliminiert werden (LINHARES, 2012; PAPA, 2013).

Die Metabolisierung von MFD in der Leber erfolgt durch die Wirkung von Carboxylesterasen (CES) - hauptsächlich CES1. Mutationen im Gen, das für das Enzym kodiert, sind mit einem Defizit im Stoffwechsel des Arzneimittels verbunden, das den Anwender anfällig für die Entwicklung von Nebenwirkungen ist (BATISTA, 2015). 


\section{SICHERHEIT DER VERWENDUNG}

Da es sich um ein Amphetamin-Derivat, MFD löst mehrere Diskussionen in der Literatur über die Fähigkeit dieses Medikaments, chemische Abhängigkeit und übermäßigen Gebrauch bei Benutzern verursachen, die immer noch umstritten ist. Die Seltenheit von Fällen chemischer Abhängigkeit und Missbrauch deutet auf ein geringes Risiko von Methylphenidat hin (BRANT; CARVALHO, 2012; FERNÁNDEZ, 2018; PALHARES, 2015; PASTURA; MATTOS, 2004).

Es wird empfohlen, dass das mögliche Vorhandensein von emotionaler Instabilität im Patienten vor der Verschreibung der MFD bewertet werden, was auf das Risiko zurückzuführen ist, dass diese Personen die Dosen des Medikaments spontan erhöhen (BRANT; CARVALHO, 2012).

Personen, die zuvor Komorbiditäten wie systemische arterielle Hypertonie, Herzrhythmusstörungen, Depressionen, Epilepsie und bipolare Störungen hatten, sollten mit Vorsicht in Bezug auf die Verwendung von MFD behandelt werden. In den ersten beiden Fällen sollten die Patienten im Laufe der Zeit überwacht werden, um mögliche Komplikationen zu erkennen. Im dritten Fall ist mfd mit Antidepressiva möglich. In den letzten beiden Fällen wird berichtet, dass es Sicherheit in Bezug auf die Verwendung von MFD gibt, vorausgesetzt, dass der Patient seinen klinischen Zustand stabilisiert und ordnungsgemäß behandelt hat (LOUZÃ; MATTOS, 2007).

Unter den verfügbaren Formulierungen des Medikaments, die Formulierung der verlängerten Freisetzung $\left(\right.$ Concerta $\left.{ }^{\circledR}\right)$, neben der praktischsten, weil es eine längere Dauer hat, ist auch die sicherste, da es das Auftreten von schädlichen Auswirkungen durch den plötzlichen Anstieg der Methylphenidat-Spiegel im Körper verhindert, ohne seine Wirksamkeit zu verlieren (FARIA, 2017; LOUZÃ; MATTOS, 2007; PAPA, 2013).

Obwohl MFD von der brasilianischen Gesetzgebung in die A3-Liste der psychotropen Substanzen aufgenommen wird, hat Methylphenidat nicht die gleiche Pharmakotoxizität wie die anderen Substanzen in dieser Gruppe (Methamphetamin, 
Phencyclidin, Dronabinol, u.a.), was ihm eine größere Sicherheit bei der Anwendung gibt (CARLINI et al., 2003).

\section{MFD-VERBRAUCH}

Seit dem dritten Jahrzehnt des 20. Jahrhunderts hat die Klasse der aus Amphetamin gewonnenen Medikamente das Interesse der pharmazeutischen Industrie für die Behandlung von Verhaltensstörungen geweckt, da sie die SNC stimulieren kann (CÂNDIDO, 2018; FERNÁNDEZ, 2018).

Die MFD wurde 1944 von Leandro Panizzon synthetisiert und 1954 unter dem Namen Ritalin (CÂNDIDO et al., 2019). Auch mit seiner Existenz, bis dahin war es nicht bekannt, dass seine Verwendung Verbesserungen am Bild von TDAH bringen könnte. Dies wurde erst in den 1960er und 1970er Jahren festgestellt, als Studien vorteile für Kinder mit Verhaltensstörungen (BRANT; CARVALHO, 2012; GONÇALVES; PEDRO, 2018).

Trotzdem, MFD Verbrauch wurde bereits in großem Maßstab weltweit etabliert, zur Behandlung von chronischer Müdigkeit und Narkolepsie, neben der Verwendung für Gewichtsverlust, Verbesserung der sportlichen Leistung und geistige Leistung (BRANT; CARVALHO, 2012; BRUNELLI, 2013). Nach Barros und Ortega (2011), nicht-therapeutische Anwendungen werden Freizeit genannt, wenn verwendet, um Wachheit Zeit und die Bereitschaft, Freizeit zu üben, ästhetische Aktivitäten, wenn für Gewichtsverlust verwendet, und kognitive Verbesserung.

In den 1960er Jahren, durch die Überprüfung der Verbesserung des klinischen Bildes von ADHS mit der Verabreichung von MFD, kam die wissenschaftliche Gemeinschaft zu einem Konsens über die Verwendung des Medikaments zur Diagnose dieser Erkrankung, die ursprünglich für Kinder etabliert wurde, aber, als weitere Studien entwickelt wurden, wurde auch für Jugendliche und Erwachsene wirksam. Wenn der Mensch mit Verdacht auf TDAH nach der Verwendung der MFD eine Verbesserung zeigte, wurde angenommen, dass er an der Krankheit litt (BRUNELLI, 2013; 
GONÇALVES; PEDRO, 2018). Dieser Aspekt hat zusammen mit der Ausweitung der Kriterien für die Diagnose von TDAH und der daraus resultierenden Zunahme der Inzidenz von TDAH in den letzten Jahren zur Verschlimmerung seines Konsums beigetragen (GONÇALVES; PEDRO, 2018; SALVIANO, 2015).

Was jedoch im aktuellen Szenario die größte Aufmerksamkeit erregt, ist die Aneignung des Verbrauchs von MFD für nichttherapeutische Zwecke, vor allem von Personen über 19 Jahren, Kurs Studenten, Konverkursien, Führungskräften, Unternehmern und Angehörigen des Gesundheitswesens als Hauptnutzer, die die Hauptmotivation für eine höhere Leistung während ihrer Aktivitäten darstellen (BRANT; CARVALHO, 2012; CALIMAN; RODRIGUES, 2014; CÂNDIDO et al., 2019; GONÇALVES; PEDRO, 2018; MORGAN et al., 2017; SALVIANO, 2015).

Es lohnt sich, die Erhöhung des Konsums bei Jugendlichen hervorzuheben, für den Einsatz im Zusammenhang mit vermindertem Appetit und Müdigkeit, zusätzlich zu Neuroenhancement (CÂNDIDO et al., 2019; GONÇALVES; PEDRO, 2018; SALVIANO, 2015). Die Studie, die von Cândido et al. (2019) fand heraus, dass von den Studenten bewertet, die Methylphenidat für Neuroenhancement verwendet, etwa ein Drittel von innen erwarb das Medikament ohne Rezept. Dieselben Autoren weisen auf die Notwendigkeit eines Eingreifens in diese Praxis hin, da es an Beweisen für die Wirksamkeit der MFD zu diesem Zweck fehlt.

\section{DOSIERUNG VON MFD UND ITS RELATIONSHIP MIT DEN NEBENWIRKUNGEN DER DRUG}

Einige Studien zeigen, dass die Entwicklung von Nebenwirkungen durch die Verwendung von MFD, wie Schlaflosigkeit und verminderten Appetit, durch die Dosierung verabreicht bedingt ist, und diese Ereignisse können im Laufe der Zeit oder mit verminderter Dosierung zurückgehen (BATISTA, 2015; BATISTELA, 2011; LOUZÃ; MATTOS, 2007; PASTURA; MATTOS, 2004). 
Holmskov et al. (2017), basierend auf einer Studie über gastrointestinale Nebenwirkungen während der Behandlung mit Methylphenidat, fand keine Unterschiede im Risiko von Nebenwirkungen, wenn Dosis, Art oder Dauer der Verabreichung berücksichtigt.

Eine geringe Häufigkeit der Entwicklung von Nebenwirkungen bei Denanwendern des Medikaments kontaktiert wird, und in den meisten Fällen sind sie mild oder mäßig (SALVIANO, 2015).

Bei Kindern erfolgt die Behandlung von TDAH mit Dosierungen von 5 bis $20 \mathrm{mg} / \mathrm{Tag}$, mit Anpassungen nach Bedarf (FARIA, 2017).

Papa ${ }^{1}$ weist darauf hin, dass keine kardiovaskulären Veränderungen mit Dosierungen unter $30 \mathrm{mg}$ gefunden werden. Dies ist wichtig für die Therapie der Krankheit, da es Berichte über kardiovaskuläre Veränderungen mit der Verwendung von MFD gibt (FARIA, 2017; HENNISSEN et al., 2017; SALVIANO, 2015).

Um die Beschwerden zu lindern, die durch einige Nebenwirkungen erzeugt werden, gibt es Empfehlungen zu befolgen: die Verwendung von MFD mit der Bildung von unerwünschten Ereignissen einzustellen, um zu überprüfen, ob das Medikament der Erreger war. In Fällen von Verschlechterung zusammen mit den Spitzenwerten der MFD im Körper, Es kann die Verwendung der Long-Release-Formulierung vorgeschlagen werden. Bei Schlaflosigkeit, Vermeidung der Verwendung von MFD in der Nähe des Schlafengehens, Erhöhung der Dosierung am Morgen und abnehmend in der Nacht, sind Maßnahmen, die wirksam sind. Für Appetitlosigkeit kann der Patient für die Kurzwirkungsformulierung entscheiden, um die Wirkungszeit des Medikaments und damit die von inm geförderte Nebenwirkung (PASTURA; MATTOS, 2004).

\section{SCHLUSSFOLGERUNG}

Die Auswirkungen von MFD stammen aus der Hemmung von NeurotransmitterRezeptoren wie Dopamin und Noradrenalin in kortikalen und subkortikalen Regionen im Zusammenhang mit motorischer Kontrolle und Aufmerksamkeit. Mit dieser RC: 84391

Zugriffsverbindung: 
Hemmung, die Neurotransmitter sind in größerer Menge der synaptischen Spalte, verursacht die Auswirkungen des Medikaments.

MFD kann chemische Abhängigkeit erzeugen, da sie aus Amphetaminen gewonnen wird, aber in der Literatur gibt es nur wenige Fälle von Abhängigkeit, die sich aus ihrer Verwendung ergeben.

MFD ist indiziert für Patienten mit TDAH diagnostiziert, kann aber auch für andere Zwecke verwendet werden, einschließlich der Behandlung von Narkolepsie, kognitive Verbesserung, Erholung, Ästhetik, ein alternativer Ansatz zur akuten Manie, und Behandlung von Symptomen von Patienten mit fortgeschrittenen Neoplasmen.

Die Verwendung von MFD kann Nebenwirkungen verursachen, von denen die wichtigsten Schlaflosigkeit und Appetitlosigkeit sind, und diese Effekte sind direkt proportional zu den Dosen aufgenommen.

Die Literatur zeigt, dass MFD bei der Behandlung von ADHS effizient ist, macht aber nicht klar, ob es Abhängigkeit verursacht, noch wird es negativ auf das Wachstum und die Gewichtszunahme von Kindern beeinflusst.

\section{VERWEISE}

BARROS, D.; ORTEGA, F. Metilfenidato e Aprimoramento Cognitivo Farmacológico: representações sociais de universitários. Saúde Soc, v. 20, n. 2, p. 350-63, 2011.

BATISTA, J. M. N. Estudo farmacoterapêutico e farmacogenético em crianças e adolescentes com transtorno do déficit de atenção e hiperatividade tratados com metilfenidato. Tese de Doutorado - Universidade de Federal do Ceará. Fortaleza, p. 95. 2015.

BATISTELA, S. Efeitos da administração aguda de diferentes doses do metilfenidato sobre a cognição de jovens saudáveis. Tese (Mestre em Ciências) - Universidade Federal de São Paulo. São Paulo, p. 88. 2011. 
BRANT, L. C.; CARVALHO, T. R. F. Metilfenidato: medicamento gadget da contemporaneidade. Interface comun saúde educ, v. 16, n. 42, p. 623-36, 2012.

BRUNELLI, L. F. O Metilfenidato (RITALINA®) na escola: Percepção dos educadores da rede de Ensino fundamental I e II. Dissertação (Mestrado em Ciências Humanas e Sociais Aplicadas) Universidade Federal de Minas Gerais. Belo Horizonte, p. 100. 2013.

CALDEIRA, C. M. P. Frequência do transtorno de déficit de atenção e hiperatividade (TDAH) e os problemas psicológicos associados em crianças e adolescentes. Monografia (Especialista em Neurociências) - Universidade de Federal do Ceará. Fortaleza. p. 64. 2015.

CALIMAN, L. V.; DOMITROVIC, N. Uma análise da dispensa pública do metilfenidato no Brasil: o caso do Espírito Santo. Physis, v. 23, n. 3, p. 879-902, 2013.

CALIMAN, L. V.; RODRIGUES, P. H. P. A experiência do uso de metilfenidato em adultos diagnosticados com TDAH. Psicol estud, v. 19, n. 1, p. 125-134, 2014.

CAMPOS, M. P. O. et al. Fadiga relacionada ao câncer: uma revisão. Rev Assoc Med Bras, v. 57, n. 2, p. 211-219, 2016.

CÂNDIDO, R. C. F. Metilfenidato de liberação imediata para o transtorno do déficit de atenção e hiperatividade (TDAH) em adultos - revisão sistemática. Dissertação (Mestrado em Medicamentos e Assistência Farmacêutica) - Universidade Federal de Minas Gerais. Belo Horizonte, p. 147. 2018.

CÂNDIDO, R. C. F. et al. Perini E, Pádua CM, Junqueira DR. Prevalência e fatores associados ao uso de metilfenidato para neuroaprimoramento farmacológico entre estudantes universitários. Psicol conoc Soc, v. 18, p. 1-7, 2019. 
CARLINI, E. A. et al. Metilfenidato: influência da notificação de receita A (cor amarela) sobre a prática de prescrição por médicos brasileiros. Rev Psiquiatr Clín, v. 30, n. 1, p. 11-20, 2003.

FARIA, J. C. M. Desempenho acadêmico de estudantes com diagnóstico de transtorno do déficit de atenção e hiperatividade (TDAH) em uso de metilfenidato - revisão sistemática. Dissertação (Mestrado em Medicamentos e Assistência Farmacêutica) - Universidade Federal de Minas Gerais. Belo Horizonte, p. 77.2017.

FERNÁNDEZ, L. C. S. Psicoestimulantes para el TDAH: análisis integral para una medicina basada en la prudencia. Rev Asoc Esp Neuropsiq, v. 38, n. 133, p. 301330. 2018.

FREESE, L. et al. Uso não médico de metilfenidato: uma revisão. Trends psychiatry psychother, v 34, n. 2, p. 110-115, 2012.

GONÇALVES, C. S.; PEDRO, R. M. L. R. "Drogas da Inteligência?”: Cartografando as controvérsias do consumo da Ritalina para o aprimoramento cognitivo. Psicol conoc Soc, v. 8, n. 2, p 71-94, 2018.

GRAZINA, R. et al. Fractura por estrés del cuello del fémur en unaniña hiperactiva tratada con metilfenidato - Reporte de caso. Rev Asoc Argent Ortop Traumatol, v. 83 , n. 1, p. 45-49, mar. 2018.

HENNISSEN, L. et al. Cardiovascular Effects of Stimulant and Non-Stimulant Medication for Children and Adolescents with ADHD: A Systematic Review and MetaAnalysis of Trials of Methylphenidate, Amphetamines and Atomoxetine. CNS drugs, v. 31, n. 3, p. 199-215, 2017.

HOLMSKOV, M. et al. Gastrointestinal adverse events during methylphenidate treatment of children and adolescents with attention déficit hyperactivity disorder: A 
systematic review with meta-analysis and Trial Sequential Analysis of randomised clinical trials. PLos ONE, v. 12, n. 6, p. 1-18, 2017.

LIANG, E. F. et al. The Effect of Methylphenidate and Atomoxetine on Heart Rate and Systolic Blood Pressure in Young People and Adults with Attention-Deficit Hyperactivity Disorder (ADHD): Systematic Review, Meta-Analysis, and MetaRegression. Int J Environ Res Health Public, v. 17, n. 1789, p 1-16, 2018.

LIMA, K. S. et al. Efeitos do metilfenidato sobre as glândulas salivares maternas de camundongos. Rev Odontol UNESP, v. 45, n. 6, p. 316-321, 2016.

LINHARES, M. I. Estudo da Ritalina ${ }^{\circledR}$ (cloridrato de metilfenidato) sobre o sistema nervoso central de animais jovens e adultos: aspectos comportamentais e neuroquímicos. Dissertação (Mestrado em Farmacologia) Universidade Federal do Ceará. Fortaleza, p. 145. 2012.

LÓPEZ-GARCÍA, P.; HEGERL, U. Tratamiento de la manía aguda com metilfenidato: propuesta terapêutica basada en un nuevo modelo fisiopatológico. Rev psiquiatr salud ment, v. 6, n. 2, p. 93-94, 2013.

LOUZÃ, M. R.; MATTOS, P. Questões atuais no tratamento farmacológico do TDAH em adultos com metilfenidato. J bras psiquiatr, v. 56, n. 1, p. 53-56, 2007.

LUDVIGSSON, M.; HAENNI, A. Methylphenidate toxicity after Roux-en-Y gastric bypass. Surg Obes Relat Dis, v. 12, n. 5, p. 55-57, 2016.

MERIDOR, K.; LEVY, Y. Systemic sclerosis induced by CNS stimulants for ADHD: A case series and review of the literature. Autoimmun Rev, v. 19, n. 1, p. 1-3, 2019.

MORAN, L. V. et al. Psychosis with Methylphenidate or Amphetamine in Patients with ADHD. N Engl J Med, v. 380, n. 12, p. 1128-1138, 2019. 
MORGAN, H. L. et al. Consumo de Estimulantes Cerebrais por Estudantes de Medicina de uma Universidade do Extremo Sul do Brasil: Prevalência, Motivação e Efeitos Percebidos. Rev bras educ med, v. 41, n. 1, p. 102-109, 2017.

NÚÑES-GARCES, M.; SÁNCHEZ-GAYANGO, A.; ROMERO-PÉREZ, C. Alopecia reversible secundaria a metilfenidato OROS. Rev colomb psiquiatr. 2018. DOI: 10.1016/j.rcp.2018.09.003.

OCHI, N. O.; VIEIRA, G. L. Efeitos do metilfenidato no desempenho motor de crianças com TDAH. Revista Saúde e Pesquisa, v. 9, n. 1, p. 93-96, 2016.

OTERO, M. I. et al. Metilfenidato y fenómeno de Raynaud secundario. Semergen, v. 39, n. 6, p. 330-334, 2012.

PALHARES, J. P. P. "Eu tomo medicamentos para estudar": compreendendo a experiência com metilfenidato entre estudantes universitários. Dissertação (Mestrado em Medicamentos e Assistência Farmacêutica - Universidade de Federal de Minas Gerais. Belo Horizonte, p. 86. 2015.

PAPA, M. P. Uso del metilfenidato en pacientes con cáncer avanzado. Rev Med Urug, v. 29 , n. 1 , p. $58-63,2013$.

PASSOS, R. B. F.; LÓPEZ, J. R. R. A. Síndrome de Gilles de la Tourette associada ao transtorno de déficit de atenção com hiperatividade: resposta clínica satisfatória a inibidor seletivo da recaptura de serotonina e metilfenidato. J bras psiquiatr, v. 59 , n. 2, p. 160-162, 2010.

PASTURA, G; MATTOS, P. Efeitos colaterais do Metilfenidato. Revista de Psiquiatria Clínica, v. 31, n. 2, p. 100-104, 2004.

RAPELLO, G. V. G. et al. Pulmonary emphysema induced by methylphenidate: experimental study. Sao Paulo Med J, v. 133, n. 2, p. 131-134, 2015. 
ROCHA, F. L.; MALLOY-DINIZ, L. F.; HARA, C. Emprego de metilfenidato para o tratamento de déficit cognitivo em paciente com seqüela de traumatismo cranioencefálico. J bras psiquiatr, v. 55, n. 1, p. 78-81, 2006.

SALVIANO, L. H. M. S. Estudo de segurança da Ritalina ${ }^{\circledR}$ (cloridrato de metilfenidato) em animais adultos: aspectos de neurotoxicidade e nefrotoxicidade. Tese de doutorado - Universidade de Federal do Ceará, Fortaleza, p. 149.2015.

Eingereicht: Mai 2021.

Genehmigt: Mai 2021. 